\title{
Epidemiología del tabaquismo en los estudiantes de ciencias de la salud*
}

\author{
Tobacco use in health-science students
}

\author{
Andres Prat-Marin ${ }^{\star \star}$, M.M. Fuentes-Almendras ${ }^{\star \star}$, P. Sanz-Gallen ${ }^{\star \star}$, R. Canela-Argues ${ }^{\star \star}, J$. \\ Canela-Soler ${ }^{\star \star}$, H. Pardell-Alenta**, L.L. Salleras-Sanmarti**
}

\begin{abstract}
PRAT-MARIN, A. et al. Epidemiología del tabaquismo en los estudiantes de ciencias de la salud. Rev. Saúde Pública, 28: 100-6, 1994. Las características del hábito tabáquico cn los cstudiantes de la División de Ciencias de la Salud de la Universidad de Barcelona han sido cstudiadas en este trabajo. Durante el Curso Académico 1988-89, 382 alumnos han sido entrevistados individualmente en relación al tabaquismo, mediante el cuestionario utilizado habitualmente por el Departamento de Sanidad y Seguridad Social de la Generalidad de Cataluña (España), con las modificaciones más oportunas para este colectivo especifico. Asinismo se ha recogido información relacionada con la inlluencia de los estudios universitarios en el hábito de fumar, la autorización de la publicidad y la eficacia de los programas y campanas de lucha antitabaco desarrollados por las Administraciones públicas. La prevalencia de tabaquismo ha sido del $40,8 \%$ (29,8\% de fumadores habituales y $11 \%$ de fumadores ocasionales). Ilay que remarcar que los estudios relacionados con la salud no parecen influir de forma destacada en el hábito de los estudiantes, siendo el entorno cultural y social los factores que tienen un papel más determinante en este sentido. Como conclusión final señalar que, para disminuir la prevalencia de tabaquismo en este importante colectivo, hay que instaurar programas educativos a nivel de la escolarización pre-universitaria (bachillerato unificado polivalente y curso de orientación universitaria), e introducir modificaciones en los futuros planes de estudio de las profesiones sanitarias, de manera que los contenidos relacionados con el tabaco sean más motivadores para los alumnos.
\end{abstract}

Descriptores: Tabaquismo, epidemiologia. Estudiantes del area de la salud.

\section{Introducción}

El consumo de tabaco es la principal causa prevenible de pérdida de salud y de muerte prematura que tienen actualmente los paises desarrollados, y representa una seria amenaza para la salud pública de los países en vías de desarrollo.

Actualmente en Europa se producen aproximadamente un millón de muertes anuales como consecuencia del tabaquismo. En la Región de las Américas se cifran en medio millón las muertes prematuras debidas al tabaco $4,5,15,25,30,38,39$.

Los profesionales sanitarios (médicos, farmacéuticos, diplomados en enfermería, e

\footnotetext{
- Trabajo realizado gracias a una ayuda de la CIRIT (Comissió Interdepartamental de Reccrca i Innovació Tecnológica).

* Departamento de Salud Publica y Legislación Sanitaria Universidad de Barcelona, España

Separatas/Reprints: A. Prat-Marin - Apartado de Correos $n^{2} 14.186$ 08080 Barcelona (España)
}

outros), constituyen el colectivo social con mayor capacidad de influir positiva o negativamente en la prevalencia del hábito de fumar en una colectividad.

Pueden tener un triple rol o papel a desempeñar en la lucha antitabáquica: modelos ejemplarizadores, educadores sanitarios y sensibilizadores del impacto del tabaco en la comunidad ${ }^{17,37}$.

El papel ejemplar o modélico es ejercido cuando los profesionales sanitarios son "no fumadores" o "exfumadores".

Actúan como agentes de educación sanitaria cuando informan al resto de la población de los riesgos sanitarios del consumo del tabaco, motivando y fomentando el abandono del hábito.

Finalmente, los profesionales sanitarios pueden desempeñar un importante papel como lícleres activos para promover la prohibición o limitación de las prácticas tabáquicas en sus lugares de trabajo, y sensibilizando sobre esta temática al resto de la comunidad. 
Diferentes trabajos publicados en nuestro medio ponen de manifiesto que los profesionales de la salud presentan unas tasas de prevalencia de tabaquismo y unas actitudes en relación a este hábito que, si bién han ido mejorando progresivamente en los últimos años, todavía no alcanzan los niveles que serían deseables $8,12,18,19,31,33,34$.

En este sentido reviste especial interés el estudio de la epidemiología del tabaquismo en aquellos grupos que se encuentran en fase de formación para la salud y que en el futuro deberán ser agentes sanitarios en sus diferentes niveles de actuación profesional.

El objetivo de este estudio es conocer la prevalencia y las actitudes relacionadas con el tabaquismo en estudiantes.

\section{Material y Metodo}

La población objeto del estudio ha estado constituida por todos los alumnos matriculados durante el Curso Académico 1988-1989 de la División de Ciencias de la Salud de la Universidad de Barcelona. La ciudad olímpica de Barcelona es la capital de Catalunya (España), posee una importante densidad de población ${ }^{26}$ y la Administración Sanitaria Autonómica tiene un Plan de Salud que contempla la lucha antitabaco como un objetivo prioritario?.

A partir de los listados proporcionados por las diferentes secretarias administrativas de la Universidad de Barcelona, se efectuó un muestreo aleatorio estratificado por Facultades: Medicina ( 6 años de carrera universitaria); Farmacia (5 años); Odontología (5 años); Psicología (5 años) y Diplomados en enfermería (3 años). Debe señalarse que cuando se realizó el estudio la Facultad de Odontología se encontraba en su segundo año de funcionamiento.

El tamaño teórico de la muestra ha sido de 400 estudiantes, con un riesgo alfa del $5 \%$ y una precisión de $\pm 5 \%$. El diseño de la investigación se corresponde con un estudio transversal de prevalencia.

El trabajo de campo fue realizado por personal especialmente entrenado en esta temática durante el mes de Abril de 1989, mediante entrevista personal.

El cuestionario de recogida de la información ha sido el emplcado habitualmente por el Departamento de Sanidad y Seguridad Social de la Generalitat de Catalunya, con las modificaciones más oportunas para este colectivo específico ${ }^{8}$.

Siguiendo las recomendaciones de la Organización Mundial de la Salud, los estudiantes se han clasificado, en relación al hábito tabáquico, en cuatro categorías ${ }^{42}$ : fumadores habituales, fumadores ocasionales, exfumadores y otros no fumadores. Fumadores habituales, son todos aquellos estudiantes que en el momento de realizar cl estudio fumaban diariamente cualquier tipo de producto derivado del tabaco. Fumadores ocasionales son aquellos que fumaban, aunque no diariamente. Exfumadores son los individuos que en el momento de la encuesta ya no fumaban, aunque habían sido fumadores diarios durante 6 ó más meses. Otros no fumadores son aquellos que nunca habian fumado, o que si habian sido fumadores en el pasado, no llegaron a fumar diariamente durante 6 ó más meses.

La comparación de proporciones se ha efectuado mediante el test del ji-cuadrado y el test exacto de Fisher cuando los efectivos esperados han sido inferiores a 5 . Se ha considerado la significación estadística con un $\alpha=0,05$. Los cálculos estadísticos se han realizado con el programa informático EPI INTO versión 5.

\section{Resultados}

De los 382 alumnos encuestados, 121 han sido varones $(31,7 \%)$ y 261 mujeres $(68,3 \%)$, con una media de edad de 22,1 $\pm 3,4$ años.

La prevalencia de fumadores actuales (Habituales + Ocasionales) ha sido del 40,8\%, representando los no fumadores (exfumadores y otros no fumadores) un $59,2 \%$ del colectivo estudiado. En la Tabla 1 se presenta la distribución de la prevalencia del hábito tabáquico según sexo y frecuencia de consumo.

No se han encontrado diferencias estadísticamente significativas con respecto al sexo para las diferentes categorías del hábito. 
Tabla 1 - Prevalencia del hábito tabáquico en estudiantes de Ciencias de la Salud, según sexo y frecuencia de consumo. Universidad de Barcelona, 1988-1989

\begin{tabular}{lcccccc}
\hline Sexo & $\begin{array}{c}\text { Fumadores } \\
\text { Habituales } \\
(H)(\%)\end{array}$ & $\begin{array}{c}\text { Fumadores } \\
(0)(\%)\end{array}$ & $\begin{array}{c}\text { Fumadores } \\
\text { Actuales } \\
(\mathrm{H}+0)(\%)\end{array}$ & $\begin{array}{c}\text { Exfuma- Tores }(\%) \\
\text { dotal }\end{array}$ \\
\hline Varones & 26,4 & 13,2 & 39,7 & 10,0 & 121 \\
Mujeres & 31,4 & 10,0 & 41,3 & 8,4 & 261 \\
Total & 29,8 & 11,0 & 40,8 & 8,9 & 382 \\
\hline
\end{tabular}

Tabla 2 - Prevalencia de fumadores actuales $(H+0)$ en estudiantes de Ciencias de la Salud según Facultad. Universidad de Barcelona, 1988-1989

\begin{tabular}{lcr}
\hline Facultad & $\begin{array}{c}\text { Fumadores Actuales }(\mathrm{H}+\mathrm{O}) \\
(\%)\end{array}$ & Tolal \\
\hline Medicina & 32,9 & 85 \\
Farmacia & 33,5 & 128 \\
Odontologia & 40,0 & 10 \\
Enfermería & 58,6 & 29 \\
Psicologia & 49,2 & 130 \\
Total & 40,8 & 382 \\
\hline $\mathrm{p}=0,01$ & &
\end{tabular}

Tabla 3 . Prevalencia de fumadores actuales $(H+0)$ en estudiantes de Ciencias de la Salud, según el nivel académico. Universidad de Barcelona, 1988-1989

\begin{tabular}{lcc}
\hline $\begin{array}{c}\text { Nivel } \\
\text { Académico }\end{array}$ & $\begin{array}{c}\text { Fumadores } A c t u a l c s \\
(\%)\end{array}$ & Total \\
\hline Primer Curso & 44,6 & 83 \\
Segundo Curso & 39,0 & 82 \\
Tercer Curso & 39,5 & 76 \\
Cuarto Curso & 37,5 & 64 \\
Quinto Curso & 42,8 & 63 \\
Sexto Curso & 42,8 & 14 \\
Total & 40,8 & 382 \\
\hline
\end{tabular}

La edad media de iniciación al hábito tabáquico se sitúa cn $\operatorname{los} 16,7 \pm 1,8$ años de edad. La prevalencia de fumadores actuales según la Facultad Universitaria se muestra en la Tabla 2, encontrándose diferencias estadisticamente significalivas cntre las diversas facultades $(p=0,01)$. No se han encontrado diferencias estadísticamente significativas entre la prevalencia de fumadores y los distintos cursos académicos (Tabla 3).
Agrupando los fumadores por el número de cigarrillos fumados habitualmentc obscrvamos que: cl $41 \%$ fuma menos de $10 \mathrm{cig} / \mathrm{di}$ ia, el $13 \%$ cntre $10-20 \mathrm{cig} / \mathrm{dia}$ y el $16 \%$ restante más de 20 $\mathrm{cig} / \mathrm{día}$.

1:1 100\% de los encuestados admiten que el fumar resulta perjudicial para la salud. Sin embargo, con respecto al grado de preocupación accrea de los alectos del tabaco sobre su salud, un $11,7 \%$ dicc estar preocupado, un $33,1 \%$ poco preocupado y un $21,8 \%$ refieren no estar preocupados al respecto. Se han evidenciado diferencias estadísticamente significaltivats en relación a la condición de fumador o no fumador $(p=0,006) \cdot \Lambda$ considerar los efectos nocivos del tabaco sobre la salud de los fumadores pasivos, un $61 \%$ de los no fumadores lo consideran muy perjudicial $(p<0,00,3)$.

Un $55,6 \%$ de los encuestados considera suficientc el tratamicnto que la temática del tabaco y la salud recibe en sus estudios de Licenciatura, no detectandose diferencias significativas en relación a la condición de fumador o no fumador.

Un $70,2 \%$ de los estudiantes considera que sus estudios universitatios no cstán influcnciando en su actitud con respecto al tabaco, siendo este hecho más evidente en los no fumadores $(70 \%)$ en comparación a los fumadores $(p<0,0001)$.

Al valorar la predisposición de los estudiantes de la División de Ciencias de la Salud como agentes de Educación Sanitaria y

Tabla 4 - Actitudes frente a la Lucha antitabáquica en los estudiantes de Ciencias de la Salud, según la categoria del hábito. Universidad de Barcelona, 1988-1989

\begin{tabular}{lccc}
\hline IIábito & $\begin{array}{c}\text { Prohibicion } \\
\text { Puldicidad } \\
(\%)\end{array}$ & $\begin{array}{c}\text { Mecliclas Legistativas } \\
\text { Restrictivals } \\
(\%)\end{array}$ & Total \\
\hline $\begin{array}{l}\text { Fumador Actual } \\
(H+0)\end{array}$ & 35,0 & 39,0 & 156 \\
No fumador & $65,0^{*}$ & $60,9^{* *}$ & 226 \\
Total & 62,8 & 85,8 & 382 \\
\hline$p<0,01$ & & & \\
$* p<0,01$ & & &
\end{tabular}


Tabla 5 - Tendencia actual de la prevalencia del tabaquismo según el Hábito de los estudiantes de Ciencias de la Salud. Universidad de Barcelona, 1988-1989

\begin{tabular}{lcccc}
\hline Hábito & $\begin{array}{c}\text { Tendencia } \\
\text { a } \\
\text { Aumentar } \\
(+) \\
(\%)\end{array}$ & $\begin{array}{c}\text { Tendencia } \\
a \\
\text { Disminuir } \\
(-) \\
(\%)\end{array}$ & $\begin{array}{c}\text { Tendencia } \\
(=) \\
(\%)\end{array}$ & $\begin{array}{c}\text { Invariable } \\
(\%)\end{array}$ \\
\hline $\begin{array}{l}\text { Fumador Actual } \\
(H+0)\end{array}$ & 37,7 & 41,9 & 41,4 & 156 \\
No Fumador & 62,2 & 58,9 & 58,5 & 226 \\
Total & 23,5 & 47,3 & 29,0 & 382 \\
\hline
\end{tabular}

desarrollar un papel activo en la lucha antitabaco, un $57 \%$ se ha manifestado positivamente el respecto; se han encontrado diferencias estadísticamente significativas en relación a la condición de no fumador $(70 \%) \mathrm{en}$ comparación a la de fumador $(p<0,0001)$.

La actitud positiva que estos estudiantes tienen respecto a la prohibición de la publicidad y las medidas legislativas de carácter restrictivo en el consumo como elcmentos de lucha antitabaco, se resumen en la Tabla 4.

En la Tabla 5, se muestia la tendencia evolutiva que la prevalencia del tabaquismo tiene en nuestro medio, en opinión de los estudiantes y según hábito de consumo.

\section{Discusión}

La prevalencia del hábito tabáquico entre los estudiantes de Ciências de la Salud de la Universidad de Barcelona $(40,8 \%)$ puede considerarse de una magnitud intermedia si se compara con estudios realizados en poblaciones similares. En el ámbito sanitario español Bobes y col. ${ }^{1}$ y el Grupo Universitario Multicéntrico ${ }^{14}$ han descrito unas prevalencias del $60 \%$ y del $71 \%$ respectivamente. Dipp y col. ${ }^{10}$ señalan una prevalencia del $50 \%$ entre estudiantes bolivianos, y Ionescu y Mihaescu ${ }^{16}$ cifra en un $67 \%$ la prevalencia entre los estudiantes de algunas profesiones sanitarias. Sin embargo, en otros estudios ${ }^{3,7,20,21,36}$ se han encontrado prevalencias inferiores a las de nuestro trabajo.

Al comparar los resultados correspondientes a las distintas Licenciaturas, sorprende la escasez de trabajos en detcrminados colectivos (farmacéuticos, odontólogos, e outros) ${ }^{15,27,28,41}$ y que muestran unos resultados equivalentes a los nuestros. Este hecho destaca la importancia de potenciar investigaciones similares entre estos futuros profesionales de la salud.

Probablemente al tener una labor clínico-asistencial más destacada, los estudios epidemiológicos realizados en estudiantes de medicina y de enfermería están más desarrollados en la literatura $1,6,12,13,14,21,22,29,40$.

Así, parece confirmarse la tendencia a un descenso progresivo de la prevalencia del tabaquismo entre los estudiantes de Medicina ${ }^{1,14,16}$, si bien nuestra prevalencia del $32,9 \%$ pucde considerarse todavía elevada.

Más alarmante es la clevada prevalencia encontrada entre los estudiantes de enfermería, y que supera ampliamente la descrita en otros trabajos sobre este colectivo ${ }^{6,13,22}$, y en diferentes profesiones sanitarios 2,35 . Hay que resaltar que una gran parte de las actividades preventivas de educación sanitaria realizadas en cl ámbito asistencial son competencia de estos importantes profesionales 32 .

Al igual que describen otros autores ${ }^{1,12}$, la impregnación tabáquica se produce antes de entrar en la Universidad, a los 15-17 años y durante los estudios de B.U.P. (Bachillerato Unificado Polivalente) y C.O.U. (Curso de Orientación Universitaria), principalmente ${ }^{11}$. Esto nos explicaría la elevada prevalencia de tabaquismo encontrada en los primeros cursos universitarios.

Por otra parte, y a pesar de la naturaleza de los estudios realizados, el grado de preocupación por los efectos del tabaco sobre la salud puede considerarse muy escaso; aunque, eso sí, la totalidad de los estudiantes admite que el fumar es perjudicial para la salud. Este resultado confirma el principio de la educación sanitaria según el cual la salud, como concepto motivador para modificar conductas insanas, es poco efectivo en personas jóvenes, ya que el problema de enfermar se percibe lejano en el tiempo ${ }^{32}$. Este hecho explicaría también que las oscilaciones en la prevalencia de tabaquismo a lo largo de los diferentes cursos de las Licenciaturas de Ciencias de la Salud no 
presente variaciones significativas. Los resultados obtenidos configuran un perfil de estabilidad y de cierto compromiso en la influencia que la carrera universitaria licne sobre el hábito de fumar en los estudiantes $12,23,24,40$.

Con independencia de ser fumador o no, no hay influencia positiva del contenido curricular en el hábito, y se considera suficiente el abordaje del tema por parte de las difcrentes materias o asignaturas que integran los respectivos planes de estudio.

En relación al potencial papel de agentes activos de educación sanilaria por parte de los estudiantes de Ciencias de la Salud, los resultados son esperanzadores. Como se ha descrito también en trabajos similares ${ }^{16,41}$, este colectivo, especialmente si es no fumador, presenta una actitud muy positiva en relación a su rol profesional y en todas aquellas actividades que configuran los frentes habituales de la lucha antitabáquica. Particularmente interesante es el resultado que señala una mayor sensibilización por los efectos nocivos del tabaco en los fumadores pasivos, por parte de los estudiantes no fumadores.

Puede considerarse optimista la percepción que los estudiantes tienen de la evolución global del tabaquismo en la sociedad, y que puede atribuirse a las sucesivas campañas que las diferentes administraciones sanitarias han llevado a cabo en los últimos años.

Finalmente quisiéramos destacar la importancia que los estudiantes de Ciencias de la Salud tienen como futuros agentes de educación sanitaria en la sociedad, y la necesidad de potenciar, en las diferentes Licenciaturas, las estrategias educativas que permitan disminuir la importante prevalencia del tabaquismo en este colectivo.

\section{Agradecimientos}

Agradecemos la colaboración del Dr. Ramón Mora de la Unidad de Bioestadística de la Facultad de Medicina de la Universidad de Barcelona por el soporte informático de este trabajo.
PRAT-MARIN, A. et al. [Tobacco use health-science students]. Rev. Salide Pública, 28: 100-6, 1994. The characteristics of tobacco use by students of the Division of IIcalth Sciences of the University of Barcclona have becn studicd. During the 1988-1989 school year, 382 students were individually interviewed about their use of tobacco by means of a questionnaire routinely used by the Department of Ilealth and Social Security of the Generalitat of Catalonia (Spain), with pertinent modifications for this specific group. These interviews were performed by appropriately trained personnel. Information was also gathered on the influence of university-level studies on smoking habits, the effect of advertising and the efficacy of antitobacco programs and campaigns carried out by the government. The study sample was drawn from lists supplied by the registrars' offices, by means of a random sampling by school (Medicine, Pharmacy, Psychology, Odontology and Nursing). The prevalence of tobacco use was $40.8 \%$ (29.8\% daily smokers and $11 \%$ occasional smokers). These results are similar to those described in the literature, although these values are somewhat higher than those in more developed countries with a longer tradition of resistance to the use of tobacco. It is important to note that studying health sciences does not appera to be a major influence on the student's habits, but that the social and cultural environment is the factor which weighs the most in this respect. In conclusion, to reduce the prevalence of tobacco use in this important group, educational programs must be begun at the pre-university level (secundary school) and changes should be made in the curricula of the health professional so that areas related to tobacco use are more motivational for students.

Keywords: Smoking, cpidemiology. Students, health occupations.

PRAT-MARIN, A. et al. Epidemiologia do hábito de fumar em estudantes de ciências da saúde. Rev. Saúde Pública, 28: 100-6 , 1994. Foram estudadas as características do hábito de fumar nos estudantes de ciências da saúde da Universidade de Barcelona. Durante o ano letivo 1988-89 foram cntrevistados 382 alunos em relação ao uso do fumo, por meio de questionário rotinamente utilizado por um Departamento daquela Universidade. no qual foram efetuadas modificações pertinentes ao grupo estudado. Foram obtidas informações relacionadas com a influência dos estudos universitários no luábito de fumar, com os efeitos da publicidade e com a eficácia dos programas c das campanhas da luta anti-fumo desenvolvidos pelas administrações públicas. A amostra foi obtida a partir das listagens fornecidas pelas diferentes secretarias admininistrativas, por meio de uma amostmgem alcatória estratificada por Faculdades (Medicina, Farmácia, Psicologia, Odontologia e Enfeimagem). A prevalência do tabagismo foi de $40,8 \%$ (29,8\% de fumantes habituais e $11 \%$ ocasionais). Os estudos relacionados com a saúde não parecem influir, de 
um forma destacada, no hábito dos estudantes, sendo o meio cultural e social o fator que exerce papel mais determinante nesse sentido. Assinala-se que, para poder diminuir a prevalência do fumo nesse importante grupo, devem-se iniciar programas educativos ao nivel de escolarização pré-universitária (bacharelato unificado polivalente e curso de orientação universitária) e introduzir modificações nos futuros planos de estudo das profissões sanitárias, de forma a que os conteúdos relacionados com o fumo sejam canais motivadores para os alunos.

Descritores: Fumo, epidemiologia. Estudantes de ciências da saúde.

\section{Referencias Bibliográficas}

1. BOBES, J. et al. Epidemiología del consumo de alcohol y tabaco en estudiantes de la Universidad de Ovícdo. Rev. Sanid. Hig. Pública, 59:381-94, 1985.

2. CASTANEDO. J. A. et al. Prevalencia del consumo de tabaco entre los profesionales sanitarios del Hospital Marqués de Valdecilla (Santander). Aten. Primaria, 4:429, 1987.

3. COE, R.M. \& CHOHEN, J.D. Clgarette smoking among medical students. Am J. Public Health 70:16971, 1980.

4. COMITE DE EXPERTOS DE LA OMS EN LA LUCHA ANTITABÁQUICA, Ginebra, 1974. Informe. Ginebra, 1975. (Série de Informes Técnicos, 568).

5. COMITE DE EXPERTOS DE LA OMS EN LA LUCHA ANTITABÁQUICA, Ginebra, 1978. Informe. Ginebra, 1979. (Série de Informes Técnicos, 636).

6. CORDÓN, F. et al. El tabaquismo en los estudiantes de enfermeria: prevalencia, actitudes y conocimientos. Gac. San., 6:5861, 1992.

7. DE COSTA, V.L. Brasil. In: Organizacion Panamericana de la Salud. Tabaco o salud: situactón en las Américas. Whashington, DC, 1992, p.74. (OPS - Publtcación Científica, 536).

8. DEPARTAMENT DE SANITAT I SEGURETAT SOCIAL. Generalitat de Catalunya. Enquesta de tabaquisme en els col. lectius exemplars de l'àmbit sanitari (metges, farmacéutics i infermeres). Informe, 1990.

9. DEPARTAMENT DE SANITAT I SEGURETAT SOCIAL. Generalitat de Catalunya. Document marc per a l'ela boractó del Pla de Salut de Catalunya. Barcelona 1991.

10. DIPP, C. et al. Uso indebido de drogas en estudiantes de Bolivia. Ed. DINAPRE, 1992.

11. FUENTES, M.M. Epidemiología del hábito tabáquico en estudiantes de enseñanza secundarta de BarcelonaCiudad 198889. Barcelona, 1990. [Tesis doctoral Departamento de Salud Pública y Legislación Sanitaria, Facultad de Medicina, Universidad de Barcelona].

12. GESTAL, J.J. \& MONTES, A. Smoking habits of final-year Galician medical students. Rev. Epidemiol Sante Publique, 35:386-92, 1987.

13. GRUPTA, A. et al. Identification of health risk factors among undergraduate university students. Nurs. Pap., 17:2447, 1985.

14. GRUPO UNIVERSITARIO MULTICETRICO. Consumo de drogas en el medio universitario. Med. Clín. (Barcelona), 75:317-26, 1980.

15. INTERNATIONAL UNION AGAINST CANCER. Guidellnes on smokting cessation. Geneve, 1984.
16. IONESCU, C. \& MIHAESCU, T. Smoking behaviour, knowledge and attitudes of medicine, dentistry and pharmacy students. Rev. Med. Chirurg., 90:7985, 1986.

17. LAPORTE, J. El papel del médico en la lucha antitabáquica. Med. Clin. (Barcelona), 89:6-9, 1987.

18. MARTÍNEZ, J. et al. Encuesta sobre el hábito tabáquico en una población de médicos hospitalarios. Rev. Clin. Esp., 182:429-34, 1988.

19. MARTÍNEZ, J.L. et al. Estudio del tabaquismo en profesionales sanitarios del medio hospitalario de Asturias. Rev. Esp. Cardiol., 43:219-26, 1990.

20. MAYNARD, $L$ et al. Behaviors and attitudes among medical students concerning cigarette smoking and alco hol consumption. J. Med. Educ., 61:921-2, 1986.

21. NACHTIGAL, D. et al. Smoking among medical and engr neering students. Med Educ, 23:196-200, 1989.

22. NEII, J.V. The smoking patterns and attitudes of student nurses and student teachers. Aust. Nurs. $J, 9: 478,1980$.

23. OCKENE, J.K. et al. A residents' training program for the development of smoking intervention skills. Arch. Intern. Med., 148:1039-45, 1988.

24. ORGANIZACIÓN MUNDIAL DE LA SALUD. Actitudes y conductas sobre el hábito de furmar en los profesionales sanitarios. Rev. San. Hig. Publica, 59:203-12, 1985.

25. ORGANIZACIÓN PANAMERICANA DE LA SALUD. Tabaco o salud: situactón en las Américas. Washington, DC, 1992. (OPS - Publicación Clentífica, 536).

26. PADRONS Municipals d'Habitants de 1991: ajuntaments de Catalunya i Consorci d'Informació i Docut mentació de Catalunya. Barcelona, 1992.

27. PIEDROLA, G. Alcohol y tabaco como contaminates sociales en el mundo technificado. Medicamenta, 314:7-18, 1974.

28. PRAT, A. et al. Epidmiología del tabaquismo en los estudiantes de Farmacia. Cir. Far., 316:273-8, 1992.

29. RIBÓ, C. et al. El tabaquismo en los estudiantes de medicina. Atenc. Prim., 9:203-6, 1992.

30. ROYAL COLLEGE OF PHYSICLANS. Smoking or health. London, Pitman Medical, 1977.

31. SAINZ, N. et al. Actitudes y conductas asociadas al consumo de tabaco en profesinales sanitarios de un hospital. Atenc. Prim., 5:81-4, 1988.

32. SALLERAS, L Educactón sanitaria: principios, métodosy aplicaciones. Madrid, Editorial Diaz de Santos, 1985.

33. SALIERAS, L. et al. Actitudes y hábitos tabáquicos de los médicos de Catalunya (España). Med. Clin. (BarceLona), 89:6-9, 1987.

34. SANCHEZ, L et al. Prevalencia del tabaquismo en la profesión médica Med. Clin (Barcelona), 90:4047, 1988.

35. SANZ, M.T. Hábitos tabáquicos: estudio en personal hospitalario. Rev. Clin. Esp., 185:391-5, 1989.

36. SUST, M. et al. El tabaquismo en los estudiantes de medicina. Med. Integral., 12:375-81, 1988.

37. The Physician's Role. Copenhagen, WHO Regional Office for Europe, 1988. (Smoke-free Europe:1).

38. TOBACCO or health: the way ahead: First European Conference on Tobacco Policy, Madrid, 1988. Copenhagen, WHO Regional Office for Europe, 1989.

39. US DEPARTMENT OF HEALTH EDUCATION AND WEL FARE. Smoking and health, a report of the Surgeon General Rockwille, 1979. (DHEW Publication nePHS 79-50066).

40. VALIESCAR, R. et al. Consumo de tabaco de la comarca de la Garrotxa: estudio comparativo 1982 - 1986. Gac. San., 24:125-9, 1991.

41. WHITLEY, R. et al. Smoking attitudes and behaviours of 
pharmacists and pharmacy students. Contemp. Pharm. Pract, 5:257-63, 1982.

42. WHO. Guidelines for the conduct of tobacco-smoking surveys among heath professionals; report of a WHO Meeting held in Winipeg, Canada, 1983. Ginebra, 1984. (WHO/SMO/84.1).

Recebido para publicação em 16.6.1993

Reapresentado em 11.1.1994

Aprovado para publicaça em 11.2.1994

\section{CONGRESSO BRASILEIRO DE EPIDEMIOLOGIA II CONGRESSO IBERO AMERICANO DE EPIDEMIOLOGIA I CONGRESSO LATINO AMERICANO DE EPIDEMIOLOGIA}

\section{PROMOÇÃO: \\ ASSOCIAÇÃO BRASILEIRA DE SAÚDE COLETIVA (ABRASCO) SOCIEDADE IBERO AMERICANA DE EPIDEMIOLOGIA (SIAE) ASSOCIAÇÄO LATINOAMERICANA DE MEDICINA SOCIAL (ALAMES) DATA E LOCAL; 24-28 DE ABRIL DE 1995, SALVADOR, BAHIA, BRASIL}

\section{TEMA CENTRAL: A EPIDEMIOLOGIA NA BUSCA DA EQÜIDADE EM SAÚDE}

\section{APRESENTAÇÃO}

A história recente da Epidemiologia mundial tem sido marcada pelo crescimento do seu papel, tanto no que diz respeito à sua capacidade de produzir conhecimentos relevantes sobre o processo saúde-doença, como para fundamentar práticas de prevenção e ações sobre os problemas de saúde.

Dentro deste contexto, no período de 24 a 28 de abril de 1995 acontecerão conjuntamente em Salvador-Bahia o III Congresso Brasileiro de Epidemtologia, o II Congresso Ibero Americano de Epidemiologia e o I Congresso Latino Americano de Epidemiologia. Acontecerá simultaneamente a 1' Mostra de Tecnologia em Epidemiologia, a qual buscará colocar em evidência os esforços e a atualidade do desenvolvimento de tecnologias em saúde coletiva. Será um dos mais importantes eventos da Epidemiologia em línguas latinas, que congregará os epidemiologistas e outros profissionais da área de saúde coletiva com o objetivo de estimular o desenvolvimento científico da disciplina e definir estratégias de conduzi-lo no esforço de transformação das condições de saúde da nossa população.

ATTVIDADES PRÉ-CONGRESSO (24-25 de abril, 1995):

\section{PROGRAMAÇÃO PRELIMINAR}

\section{OFICINAS DE TRABALHO}

- Descentralizacão e estratégias de controle de endemias. - Informações epidemiológicas nos processos decisónios em nível local. - Estado atual e tendências do ensino da Epidemiologia na pós-graduação. - Investigação cm Epidemiologia. - Programa Nacional de Capacitação em Epidemiologia - Bases da Epidemiologia Social. - Métodos epidemiológicos em doenças crônicas não transmissíveis. - Técnicas rápidas de estimativa. - Métodos de biologia molecular para investigações epidemiológicas. - Métodos epidemiológicos em saúde mental. - Epidemiologia da AIDS. - PROGRAMA DO CONGRESSO

\section{ABERTURA}

(26 de abril de 1995, 19:00 hs).

\section{CONFERÊNCIAS:}

- A Epidemiologia na busca da eqüidade em saúde. - Condições de vida e saúde no contexto das transformações em curso na América Latina - Violência e cidadania - por uma nova ética de vida - Vida e saúde nas cidades

MESAS - REDONDAS:

- Impacto das políticas econômicas e sociais nos perfis epidemiológicos - Ineqüidade em saúde - Avanços metodológicos em Epidemiologia - Investigação epidemiológica na Ibero América-Epidemiologia e gestão dos serviçōs de saúde - Ensino da Epidemiologia na América Latina.

PALESTRAS, PAINÉIS, COMUNICAÇÕES COORDENADAS, POSTERS

IMPORTANTE: OS TRABALHOS CIENTÍFICOS DEVEM SER ENTREGUES ATE 30 DE NOVEMBRO DE 1994. SOLICITAMOS AOS INTERESSADOS PARA ENVLAR SUGESTOÕES.

\section{PARA MAIORES INPORMAÇÕES}

CONGRESSO DE EPIDEMIOLOGIA - DEPARTAMENTO DE MEDICINA PREVENTTVA/UFBa

RUA PADRE FEIJÓ, 29, CANELA - 40110-170 SALVADOR, BAHIA, BRASIL

FONE: (55 71) 245-0151/245-0544/245-9003 - FAX: (55 71) 237-5856

CORREIO ELETROONICO: EPIDEMIOQSURNP. UFBA-BR 